Tivadar Feczkó, Krisztián Samu, Klára Wenzel, Branko Neral, Bojana Voncina:Textiles screen-printed with photochromic ethyl cellulose-spirooxazine composite nanoparticles COLORATION TECHNOLOGY 129(1): 18-23 (2013)

DOI: $10.1111 / \mathrm{j} .1478-4408.2012 .00404 . \mathrm{X}$ 


\section{Textiles screen-printed with photochromic ethyl cellulose-spirooxazine composite nanoparticles}

Tivadar Feczkó ${ }^{\mathrm{a}, \mathrm{b}^{*}}$, Krisztián Samu ${ }^{\mathrm{c}}$, Klára Wenzel ${ }^{\mathrm{c}}$, Branko Neral ${ }^{\mathrm{d}}$, Bojana Voncina ${ }^{\mathrm{d}}$

${ }^{a}$ Institute of Materials and Environmental Chemistry, Research Center for Natural Sciences, Hungarian Academy of Sciences, Pusztaszeri u. 59-67., H-1025, Budapest, Hungary

${ }^{\mathrm{b}}$ Research Institute of Chemical and Process Engineering, Faculty of Information Technology, University of Pannonia, Egyetem u. 10., Veszprém, 8200, Hungary

${ }^{c}$ Budapest University of Technology and Economy, Department of Mechatronics, Optics and Engineering Informatics, Faculty of Mechanical Engineering, Müegyetem rkp. 3-9., H-1111 Budapest, Hungary

${ }^{\mathrm{d}}$ Institute of Engineering Materials and Design, Faculty of Mechanical Engineering, University of Maribor, Smetanova Ulica 17, SI-2000 Maribor, Slovenia

*Corresponding author: e-mail: tivadar.feczko@gmail.com

\section{Summary}

Photochromic compounds change colour due to exposure to light while the reversion may be due either to radiation or may be thermal. The use of photochromism on fabrics can provide new opportunities to develop smart textiles, e.g. sensors and active protective clothes. Ethyl cellulose-1,3-Dihydro-1,3,3,4,5 (and 1,3,3,5,6)-pentamethyl-spiro-[2H-indole-2,3'$[3 \mathrm{H}]$ naphtha[2,1-b][1,4]oxazine] composites were prepared by an oil-in-water emulsion, solvent evaporation method in order to form easily suspendable and fatigue resistant photochromic nanoparticles in screen printing paste. Their size was well below $1 \mu \mathrm{m}$ and did not change substantially in a wide range of dye concentration. After screen printing a homogenous photochromic layer was built on a cotton substrate surface, which represented substantial blue colour development in CIELAB colour space measurements due to UV light even at a dye concentration of $0.045 \% \mathrm{w} / \mathrm{w}$. The addition of a photodegradation inhibitor, Tinuvin 144 further increased the colouration of the printed fabric.

\section{Introduction}


Photochromic materials can change colour reversibly due to stimulation by electromagnetic radiation. They are of great importance in both science and industry, being applied in ophthalmic lenses, rewritable optical recording media, data memory, optical switches, and sensors [1]. Spirooxazines (SOs) are a thoroughly studied group of photochromic dyes, which colourize (merocyanine form) owing to UV irradiation and rapidly fade back to colourless form, when the activating radiation is removed. Spirooxazines have excellent resistance to photodegradation, which has led to their various applications.

Textiles that produce adequate responses and are able to modulate the protective degree as a consequence of the external stimulus (change of UV intensity, temperature, pressure, electrical field, etc.) are called smart or intelligent textiles. An example of intelligent textiles could be photochromic textiles which change their colour owing to light [2]. The use of photochromism in textiles can provide new opportunities to develop smart garments capable of blocking UV radiation, sensing environmental changes [3], as well as design effects, or functional effects such as camouflage, security printing and brand protection [4]. Textile based sensors and active protective textiles have the advantages that their textile structure is easily customizable by sewing, thermal bonding or glueing. Further benefits are easy maintaining (washing, chemical drying), extremely large specific surface and low specific weight with improved strength, tensibility and elasticity. Workability without changing the production technology, possible integration of these types of sensors into systems of protective clothes in addition to their price and availability are also substantial advantages $[2,5]$. Cotton is the most commonly used material for woven and knitted clothing. However, due to its low weight, cotton clothing does not have a sufficient protective function against UV radiation without subsequent treatment with UV blockers. The colour of a fabric plays an important role in UV protection in the samples with a sufficiently closed surface. Especially black and blue colours exhibit high absorbance in the UV wavelength range and can provide excellent protection [6].

There are comparatively few reports of the application of photochromic compounds on textiles [4]. Photochromic textiles have been prepared by conventional dyeing techniques that applied photochromic dyes in dispersed form in exhaust dyeing $[4,7,8]$ and in screen-printing [9-11], or by attaching microcapsules containing photochromic dyes onto textile surfaces [12]. Nanomaterials such as particles, fibres, tubes or sheets coated on a substrate may display novel functionalities to the substrate because of the structure and size effect [13]. 
The commercialization of photochromic textiles requires the following main criteria to be fulfilled [14]: quick colouration and decolouration over a large temperature range, appropriate colourability and fatigue resistance.

Textile printing is the most versatile and important method used to introduce colour and design onto textile fabrics [15]. Screen printing is the most frequently applied method in textile prints. The main factors for its widespread use are the quality of the prints, applicability to almost every kind of fibre or mixture, and the ability to withstand any washing processes after fixation [16].

In our recent work [17] we found out that ethyl cellulose and poly(methyl methacrylate) are excellent media to enhance the lifetime of SO dye, and Tinuvin 144 hindered amine light stabilizer can further significantly improve the fatigue resistance of photochrome in these polymeric matrices (study under review). We found [17] that SO microencapsulation by ethyl cellulose can increase the photochromic half-life by about an order of magnitude, and this lifetime can further increase by a factor of 1.5 with using Tinuvin 144. However, poly(methyl methacrylate)-spirooxazine (PMMA-SO) had a substantially longer lifetime than ethyl cellulose-spirooxazine photochromic nanoparticles when both contained Tinuvin 144; colouration and decolouration of PMMA-SO were several times slower, which is disadvantageous in most of the application. Thus, in the present study we investigate the potential application of ethyl cellulose-spirooxazine (EC-SO) photochromic nanoparticles in screen printing of cotton fabric. As shown recently [17] these photochromic nanoparticles are much more resistant to photodegradation than the solution of the spirooxazine. In our current study, the photochromic behaviour of textile substrate screen printed with EC-SO nanoparticles containing paste was compared only with fabric screen printed with paste comprising EC-SO nanoparticles including also Tinuvin 144 hindered amine light stabilizer, which was added in order to further improve the photostability of the photochromic behaviour. The performance of photochromic textile substrates was evaluated by using colour measurements.

\section{Experimental}

\section{Materials}


Dichloromethane, polyvinyl alcohol $\left(\mathrm{M}_{\mathrm{w}}=30,000-70,000,87-90 \%\right.$ hydrolysed $)$, and ethyl cellulose (viscosity: $4 \mathrm{mPa} \mathrm{s}, 5 \mathrm{wt} \%$ in 80:20 toluene/ethyl alcohol, $25{ }^{\circ} \mathrm{C}$ ) were obtained from Sigma Aldrich. 1,3-Dihydro-1,3,3,4,5 (and 1,3,3,5,6)-pentamethyl-spiro-[2H-indole2,3'-[3H]naphtha[2,1-b][1,4]oxazine] (SO) was provided by PPG Industries. Bis $(1,2,2,6,6-$ pentamethyl-4-piperidinyl)-[[3,5-bis(1,1-dimethylethyl)-4-hydroxyphenyl]methyl]butylmalonate (Tinuvin 144) were purchased from BASF (Germany).

The textile was $100 \%$ cotton fabric, supplied by MTT Maribor (Slovenia), which was desized, scoured, bleached and mercerized. Fabric specifications were: weight $177.77 \mathrm{gm}^{-2}$, warp 27 threads $\mathrm{cm}^{-1}, 295$ tex, weft 27 threads $\mathrm{cm}^{-1}, 295$ tex and with fabric stiffness of $23.92 \mathrm{cN} \mathrm{cm}^{2}$. No further pretreatment was necessary. The printing paste was composed of Tubivis DL-650 synthetic thickener (polyacrylate dispersion fully neutralized), non-ionic Tubigat AFR-20 emulsifier (a combination of emulsifier, additives and melamine resin fixing agent), and Tubifast BN-35 binder (a thermally self-crosslinking butadiene copolymer dispersion binding agent). All paste ingredients were supplied by Bezema AG. (Switzerland).

\section{Preparation of photochromic nanocapsules}

The photochromic nanocapsules were synthesized by an oil-in-water emulsion, solvent evaporation method [17]. Briefly, 40-70 mg EC, 0-60 mg SO and in the case of further improved photochromic nanocapsules $20 \mathrm{mg}$ Tinuvin 144 were added into $4 \mathrm{ml}$ dichloromethane (DCM) stirred magnetically until complete dissolution and the formation of a clear organic phase. Oil-in-water emulsion was formulated by sonication of the organic phase in $10 \mathrm{ml}$ distilled water containing $1 \%$ polyvinyl alcohol as emulsifier using a probe sonicator, Model W-220 (Heat Systems-Ultrasonics, Inc) at $70 \mathrm{~W}$, for $60 \mathrm{~s}$ in an ice bath. In order to solidify the nanoemulsion, the DCM was removed from the emulsion droplets by evaporation during magnetic stirring for $2 \mathrm{~h}$ under atmospheric pressure at room temperature.

\section{Preparation of the printing pastes, printing and fixation process}

The stock pastes for thermal-curing were prepared from demineralized water, $3.5 \% \mathrm{w} / w$ of synthetic thickener, $2.5 \% w / w$ of emulsifier and $10 \% w / w$ binder. Printing pastes were prepared by mixing the stock paste and $1.5 \% \mathrm{w} / \mathrm{w}$ of nanoparticle dispersion consisted of distilled water and $10 \% \mathrm{w} / \mathrm{w}$ photochromic nanocapsules. Spirooxazine concentration was 30 
and $60 \% w / w$ in the nanoparticles. More fatigue resistant photochromic nanocapsules contained $30 \% w / w$ SO and $20 \% w / w$ hindered amine light stabilizer.

The printing pastes were well homogenized and applied to the cotton fabric using a laboratory flat screen printing machine Zimmer VP-RSF (Austria) with a printing screen of 70 mesh, squeegee diameter of $12 \mathrm{~mm}, 1 \mathrm{~m} \mathrm{~min}^{-1}$ printing speed, pressure grade of 1 and at two squeegee wipes. Prints were produced by printing once and then overprinting three times. Printed textiles were cured by a laboratory dryer Mathis DHE 43687 (Switzerland) with 150 ${ }^{\circ} \mathrm{C}$ hot air for $5 \mathrm{~min}$.

\section{Measuring methods}

The size distribution of photochromic nanoparticles was measured by dynamic light scattering using a Zetasizer 3600 (Malvern Instruments, Malvern, UK). The average particle size was expressed in volume mean diameter. UV-vis absorption spectra of the ethyl cellulosespirooxazine nanoparticles dispersed in distilled water were measured by Biochrom 4060 spectrophotometer (Pharmacia LKB).

The paste add-on of dried prints was determined in accordance with SIST ISO 3801:1996. The cured samples were subjected to stiffness measurements which were evaluated according to the DIN 53362:2003 method. Textile thickness was determined by SIST EN ISO 5084:1999.

Photochromic textile samples were irradiated with an OSRAM ULTRA-VITALUX® lamp of $300 \mathrm{~W}$ as a UV source, equipped with a special bulb emitting mixed light which is generated by a high-pressure lamp and a tungsten filament [18]. The ULTRA-VITALUX ${ }^{\circledR}$ lamp was produced primarily for technical applications such as curing special adhesives and plastics as well as sunlight simulation in industrial material testing. The UV source was positioned at a distance of $0.74 \mathrm{~m}$ from the surface of the fabrics. Its illuminance level was measured by a photoelement (F102, No. 853) and an ammeter (U16 No 640221).

The reflectance measurements of the printed textiles were carried out using a Minolta CM $3610 \mathrm{~d}$ reflection spectrophotometer with measurement wavelength range from $360 \mathrm{~nm}$ to 740 $\mathrm{nm}$, with the calibration reference of CM-A145 (SN: 7004502$) . \mathrm{L}^{*}, \mathrm{a}^{*}, \mathrm{~b}^{*}$ and $\Delta \mathrm{E}_{\mathrm{a}, \mathrm{b}}^{*}$ values were used to express the colour measurement results, and were computed using illuminant D65. After UV irradiation, the measuring window of the spectrophotometer was placed immediately onto the fabric sample. Since the decolouration is a thermal process, the textile 
samples were cooled below $0{ }^{\circ} \mathrm{C}$ during the UV irradiation by placing a cooler block under them. At a low temperature the thermal decay becomes very slow, thus the duration of the measurements of some seconds did not have considerable influence on it. UV irradiation was applied for $1 \mathrm{~min}$, which was sufficient to develop the full colour. The prints faded back completely to their original colour over a few minutes. After UV irradiation each sample appeared reasonably uniform visually. The photochromic colour build-up measurements were repeated 4 times, giving a standard deviation smaller than $5 \%$.

\section{Results and discussion}

\section{Size of photochromic nanoparticles}

The size measurement of ethyl cellulose-spirooxazine nanoparticles showed that the incorporation of photochromic dye supported the emulsification of nanodroplets, thus resulted in slightly smaller sizes from $5 \%$ to $15 \% w / w$ dye content than without the dye (Table 1 ). The encapsulation of higher amount of photochromic dye (30\%- $60 \% \mathrm{w} / w)$ and the addition of Tinuvin 144 light stabilizer increased the particle diameter to a small extent. It is noted that in all of the samples the maximum particle size did not exceed $530 \mathrm{~nm}$ which enables the homogenous dispersion of nanoparticles in a screen printing paste.

\section{Spectral characteristics of UV source and photochromic nanoparticle dispersions}

The illuminance level of the UV source was found to be $4500 \mathrm{~lx}$ at the applied distance of $0.74 \mathrm{~m}$. According to the producer the radiation composition of the ULTRA-VITALUX® lamp is constructed to simulate the effect of natural sunlight. However, its spectral power distribution is mostly composed of some sharp peaks in the UV and visible range [19].

The UV-vis spectra of EC-SO nanocapsules (Figure 1) were measured after the nanocapsule suspensions containing $1 \% \mathrm{w} / v$ nanocapsules were diluted to 1:200 with distilled water. The nanocapsules contained $0-60 \% w / w$ of SO related to the total capsule mass (Figure 1a). During the textile printing paste preparation, we used nanoparticle dispersions with 30 and 60 $\% w / w$ of SO, respectively, as well as a suspension containing $30 \% w / w$ SO and additionally $20 \% w / w$ Tinuvin 144. Their UV-vis spectra indicated that co-encapsulation of hindered amine light stabilizer enhances the absorption and scattering of nanoparticles substantially in 
the whole UV-vis range (Figure 1b). It is strongly emphasized that dispersions being measured, a significant proportion of their 'UV-vis absorption' results from the light scattering of nanoparticles. Thus, when we measure the absorbance spectra, it also includes scattering. In order to estimate the extent of light scattering, the UV-vis spectrum of blank capsules (particles without SO) was also studied. It can be observed that (Figure 1a) blank nanoparticles have a peak mainly in the UV region, which can be considered as a consequence of light scattering by the nanoparticles, while the absorption of the polymer plays only a minor role. It is also emphasized, by increasing the concentration of photochromic dye, not only the absorbance in the UV region, but also the scattering of nanocapsules in the visible region is enhanced. This phenomenon cannot be explained by size effect only, because size change is not considerable, while the SO concentration is varied (Table 1), but concentration increase of photochromic dye enhances the turbidity of particles, which may generate the proportional change of light scattering (Figure 2a).

SO concentration against apparent absorbance at a fixed wavelength in the UV $(304 \mathrm{~nm})$ is plotted in Figure 2b. Previously [17] we found that the increase of dye concentration resulted in a linear change of absorption in EC photochromic nanocapsules containing another SO (5chloro-1,3-dihydro-1,3,3-trimethylspiro[2H-indole-2,3'-(3H)naphth[2,1-b](1,4)oxazine]) in a wide range from 0 to $30 \% w / w$ SO related to the total capsule mass. Surprisingly, this linearity was found for the SO studied in the present work (1,3-Dihydro-1,3,3,4,5 (or 1,3,3,5,6)-pentamethyl-spiro-[2H-indole-2,3'-[3H]naphtha[2,1-b][1,4]oxazine]) in the whole analysed range of $0-60 \% \mathrm{w} / \mathrm{w}$, which means that there was no substantial dye aggregation in the capsules.

\section{Characteristics of prints containing photochromic nanocapsules}

In preliminary experiments we investigated the concentration of SO nanocapsules necessary for minimal background colour and maximal photochromic colour change of the printed textile under UV irradiation. We found that $1.5 \% \mathrm{w} / \mathrm{w}$ nanoparticles dispersion having $10 \%$ $w / w$ nanocapsules containing $30 \% w / w$ SO provided intense blue colour, and 4 layers of printing supplied a homogeneous colour coating on cotton. Consequently, the SO concentration of the printing paste was $0.045 \% w / w\left(1.26 \times 10^{-3} \mathrm{~mol} \mathrm{~L}^{-1}\right)$ which is in the range that was used by Little et al. [9-11] in their comprehensive studies. As a comparison we also investigated the application of photochromic nanocapsules containing a double amount 
$(60 \%)$ of SO which resulted in a concentration of $0.09 \% w / w\left(2.52 \times 10^{-3} \mathrm{~mol} \mathrm{~L}^{-1}\right)$ in the printing paste. In our very recent work (under review) we selected from an antioxidant, a UV absorber and 4 different hindered amine light stabilizers, Tinuvin 144 as the most efficient compound in increasing fatigue resistance. Tinuvin 144 hindered amine light stabilizer is a free radical scavenger which also contains an antioxidant moiety of the sterically hindered phenol type. It was co-encapsulated with the SO $(30 \% \mathrm{w} / \mathrm{w})$ in ethyl cellulose nanoparticles in a concentration $20 \% \mathrm{w} / \mathrm{w}$, that is $0.03 \% \mathrm{w} / \mathrm{w}\left(4.38 \times 10^{-4} \mathrm{~mol} \mathrm{~L}^{-1}\right)$ in the printing paste beside $0.045 \% w / w\left(1.26 \times 10^{-3} \mathrm{~mol} \mathrm{~L}^{-1}\right) \mathrm{SO}$.

The prepared photochromic pastes were printed on cotton fabrics using a flat bed screen printing machine, and then thermally polymerized. The paste add-on, print thickness and stiffness were determined as an average of 5 measurements and are shown in Table 2. As expected, printing increased the stiffness by about $50 \%$, and there was no significant difference in paste add-on and stiffness values among the printed fabrics containing different concentrations of photochromic dye and light stabilizer. Certainly, the thickness of textile was slightly increased due to printing. The solid mass content of printing pastes was found to be around $97 \%$ in all of the cases (Table 3 ).

\section{Evaluation of the photochromic fabrics}

Cotton fabric printed with microencapsulated photochromic dye containing paste converted reversibly from white to blue under UV irradiation. Our measurements were carried out using a colour measurement instrument and a separate UV source. The colour build-up was evaluated from reflectance measurements (Figure 3) converted to $\mathrm{L}^{*}, \mathrm{a}^{*}, \mathrm{~b}^{*}$ values using the CIELAB colour space (Table 4). Lightness ( $\left.\mathrm{L}^{*}\right)$ was the highest at the smallest SO concentration $(0.045 \% w / w)$, and its spectral reflectance was especially high in the range of 500-620 nm, where the eyes are most sensitive to light. Blueness ( $\left.b^{*}\right)$ was strong in all the three samples; as expected it was higher when the photochromic dye concentration was doubled. However, interestingly it was most pronounced using the lower concentration of SO and Tinuvin 144 light stabilizer. It is in good agreement with the finding of Little et al. [11], who also found that Tinuvin 144 enhanced not only the photochromic lifetime of spirooxazine significantly in a pigment printing method, but also the photocolouration on printed fabric.

To illustrate the colour change, the colour differences $\left(\Delta \mathrm{E}_{\mathrm{a}, \mathrm{b}}{ }\right)$ between the fabrics, printed with two different concentrations of SO and with SO and Tinuvin 144, were also determined. 
The colour differences between fabrics printed with $0.045 \% w / w$ and $0.09 \% w / w$ SO, respectively, was 4.26 ; with $0.045 \% w / w$ SO and $0.045 \% w / w$ SO plus $0.03 \% w / w$ Tinuvin 144, respectively, was 4.49, with $0.09 \% w / w$ SO and $0.045 \% w / w$ SO plus $0.03 \% w / w$ Tinuvin 144, respectively, was 2.15 . These colour differences are hardly visible even under ideal visibility conditions [20]. These results clearly indicate that by doubling the SO concentration we could not reach substantial colour development similarly to the findings of a recent study of Little et al. [11], in which authors showed that spirooxazine reaches a maximum colour development at a concentration of ca. $0.025-0.05 \% w / w$ in screen printing with disperse dye. They suggest 3 possible explanations for this phenomenon. First is the solubility limit of the dye in the binder system, which cannot occur in our system due to the microencapsulation of SO in well-dispersable nanocapsules. Their second reason, dye aggregation, is also excluded in our system as shown above. We also assume their third interpretation for our system, which attributes it to the UV self-absorption by merocyanine, which is increased with growing dye content, and limits the SO conversion in lower layers in the printed film.

\section{Conclusions}

Ethyl cellulose nanoparticles containing spirooxazine and optionally Tinuvin 144 light stabilizer can be excellently dispersed in printing paste. The spirooxazine content of nanocapsules can be varied in an extremely wide range, while their submicron size is not influenced substantially. Furthermore, their small size may also allow their use in inkjet inks, which are particularly sensitive to particle size due to small nozzle orifice. Nanoparticles were found to keep their photochromic properties in the high temperature printing process, and they did not influence the physical properties of printing paste. $0.045 \% \mathrm{w} / \mathrm{w}$ spirooxazine content related to the paste mass provided sufficient colouration, while its doubling did not further the colour development. The incorporation of Tinuvin 144 hindered amine light stabilizer slightly increased the colouration of printed fabric.

\section{Acknowledgements}

This work was granted by the Hungarian Economic Development Centre (MAG Zrt.), Hungarian Scientific Research Fund (OTKA) and EU FP7 (Marie Curie action) co-funded 
'MOBILITY' programme, reference number: OMFB-00123/210. We also acknowledge the support of the National Development Agency in Hungary (grant TÁMOP-4.2.2/B-10/1-20100025).
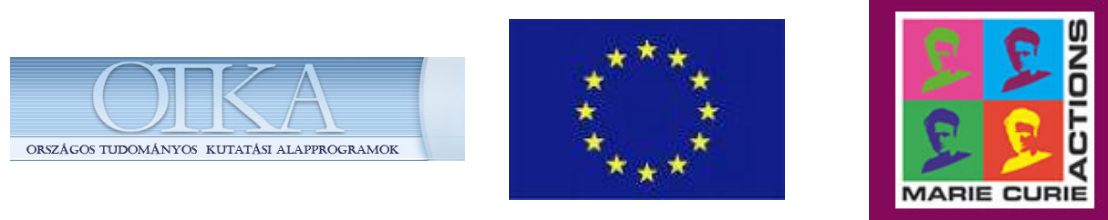

\section{References}

1. Organic Photochromic and Thermochromic Compounds, Vol. 1 Eds C J C Crano and R J Guglielmetti (New York: Kluwer Academic/Plenum Publishers, 1999).

2. M Viková, AIC 2004 Color and Paints, Interim Meeting of the International Color Association, Porto Alegre, Brazil, (2004) 130.

3. T Cheng, T Lin, R Brady and X Wang, Fiber. Polym., 9 (2008) 301.

4. S M R Billah, R M Christie and K M Morgan, Color. Technol., 124 (2008) 229.

5. M Vikova' and M Vik, Mol. Cryst. Liq. Crys., 431 (2005) 103.

6. H Gabrijelcic, R Urbas, F Sluga, K Dimitrovski, Fibres Text. East. Eur., 17 (2009) 46.

7. S M R Billah, R M Christie and R Shamey, Color. Technol., 124 (2008) 223.

8. M Aldib and R M Christie, Textile applications of photochromic dyes. Part 4: application of commercial photochromic dyes as disperse dyes to polyester by exhaust dyeing. Color. Technol. 127 (2011) 282.

9. A F Little and R M Christie. Color. Technol., 126 (2010) 157.

10. A F Little and R M Christie. Color. Technol., 126 (2010) 164.

11. A F Little and R M Christie. Color. Technol., 127 (2011) 275.

12. M Kamata, O Sasaki, S Suefuku and T Maeda, EP19910114585 (1992).

13. T Lin, X Wang, Int. J. Nanotechnol., 6 (2009) 579.

14. C Salemi-Delvaux, E Pottier, R Guglielmetti, R Dubest, J Aubard, Dyes Pigments, 40 (1999) 157.

15. R Fijan, M. Basile, R Lapasin, S Šostar-Turk, Carbohyd. Polym. 78 (2009) 25.

16. B Neral, S Sostar-Turk, B Voncina, Dyes Pigments 68 (2006) 143.

17. T Feczkó, O Varga, M Kovács, T Vidóczy and B Voncina, J. Photoch. Photobio. A., 222 (2011) 293. 
18. http://www.svetila.com/en/ultra-vitalux-300w-e27-1170.html.

19. http://www.mercateo.com/pdf/SIL/osspec-ultramed.pdf

20. J Schanda, Á Stahl, G. Lukács, XXVII. Kolorisztikai Szimpózium, Tata, Hungary, (1999) 7. 